\title{
Bifidobacterium-Rich Fecal Donor May Be a Positive Predictor for Successful Fecal Microbiota Transplantation in Patients with Irritable Bowel Syndrome
}

\author{
Shinta Mizuno ${ }^{a}$ Tatsuhiro Masaoka $^{a}$ Makoto Naganuma ${ }^{a}$ Taishiro Kishimoto $^{b}$ \\ Momoko Kitazawac Shunya Kurokawa ${ }^{\mathrm{b}}$ Moeko Nakashima ${ }^{\mathrm{a}}$ Kozue Takeshitaa, d \\ Wataru Suda $^{d, f}$ Masaru Mimura $^{b}$ Masahira Hattori ${ }^{e, f}$ Takanori Kanai $^{a}$ \\ a Division of Gastroenterology and Hepatology, Department of Internal Medicine, ${ }^{b}$ Department of Psychiatry, \\ 'Department of Ophthalmology, and d Department of Microbiology and Immunology, Keio University School of \\ Medicine, and ${ }^{\mathrm{e}} \mathrm{Graduate}$ School of Advanced Science and Engineering, Waseda University, Tokyo, and \\ ${ }^{f}$ Laboratory of Metagenomics, Graduate School of Frontier Sciences, University of Tokyo, Chiba, Japan
}

\section{Keywords}

Irritable bowel syndrome $\cdot$ Fecal microbiota

transplantation · Bifidobacterium · Dysbiosis .

Intestinal microbiota

\begin{abstract}
Background/Aims: Dysbiosis is associated with various systemic disorders including irritable bowel syndrome (IBS). Fecal microbiota transplantation (FMT) might restore intestinal microbial balance. The study aimed to determine the safety and efficacy of FMT in IBS patients, as well as also positive predictors for FMT. Methods: This was a single-arm, openlabel study. Eligible patients were diagnosed based on Rome III Diagnostic Criteria. Fecal materials were administered to the patient via colonoscopy. The primary end point was a change in the Bristol stool form scale at 4 weeks after FMT. Recovery to types 3-4 was considered a clinical response. The secondary end point was a change in intestinal microbiota and psychological status using the Hamilton Rating Scale. Results: Ten patients were enrolled. Six patients achieved a clinical response. The diversity of patients 4 weeks after FMT increased significantly compared with patients be-
\end{abstract}

\begin{tabular}{ll}
\hline KARGER & $\begin{array}{l}\text { ( ) 2017 The Author(s) } \\
\text { Published by S. Karger AG, Basel }\end{array}$ \\
$\begin{array}{l}\text { E-Mail karger@karger.com } \\
\text { www.karger.com/dig }\end{array}$ & $\begin{array}{l}\text { This article is licensed under the Creative Commons Attribution- } \\
\text { NonCommercial-NoDerivatives 4.0 International License (CC BY- } \\
\text { NC-ND) (http://www.karger.com/Services/OpenAccessLicense) } \\
\text { Usage and distribution for commercial purposes as well as any dis- } \\
\text { tribution of mons }\end{array}$
\end{tabular}

Takanori Kanai, MD, $\mathrm{PhD}$

Division of Gastroenterology and Hepatology

School of Medicine, Keio University

35 Shinanomachi, Shinjuku-ku, Tokyo 160-8582 (Japan)

E-Mail takagast@ keio.jp

\section{Introduction}

Functional gastrointestinal disorders are characterized by the presence of symptoms and the absence of organic and structural abnormalities that readily explain the symptoms. Irritable bowel syndrome (IBS) is a func- 
tional gastrointestinal disorder with symptoms thought to originate from the middle or lower gastrointestinal tract. Because its diagnosis is based only on symptoms, its etiology and pathogenesis are thought to be heterogeneous. Therefore, despite its high prevalence, established therapeutic options are still lacking. Moreover, the symptoms of IBS affect patient quality of life, and thus, IBS is a substantial cost burden to society $[1,2]$.

Recent studies reported that the composition of intestinal microbiota in IBS patients differed from that in healthy controls $[3,4]$. Changes in intestinal environment induce a compositional imbalance of microbiota, termed "dysbiosis," which is associated with IBS $[5,6]$. Current studies on microbiota gut-brain interactions demonstrated that compositional changes in the intestinal microbiota are associated with relevant abnormal gastrointestinal and brain-gut axis functions in IBS patients $[7,8]$. Visceral hypersensitivity is thought to be a pathophysiological feature in IBS that induces intensified signals from the gastrointestinal tract to the brain. This signal pathway leads to the augmentation symptomatic response in patients with IBS. A recent study suggested that intestinal microbiota and psychological distress at work were mediators of visceral hypersensitivity [9]. Based on these findings, there have been many attempts to improve IBS symptoms by the manipulation of intestinal microbiota. The administration of probiotics is the traditional treatment for dysbiosis. Treatment with $\mathrm{Bac}$ teroides infantis 35624 alleviated symptoms in IBS accompanied with changes in the cytokine profile [10]. Fermentable oligosaccharides, disaccharides, monosaccharides, and polyols (FODMAPs) can induce IBS symptoms and low FODMAP diets improve IBS symptoms $[11,12]$. Recently, McIntosh et al. [13] showed that low FODMAP diets altered the metabolome in IBS patients and increased Actinobacteria richness and diversity. However, there are some concerns with this approach because dysbiosis might be induced when using antibiotics to treat IBS. Previous clinical trials have reported the beneficial effect of probiotics in IBS patients, but efficacy was limited. Human feces contain more varied bacteria than probiotics. Therefore, human fecal microbiota transplantation (FMT) may be a candidate treatment to improve dysbiosis in patients with IBS. In recurrent Clostridium difficile infections, FMT had a higher cure rate than standard antibiotic treatment [14], and previous reports showed that FMT might restore intestinal microbial balance in human diseases [15].

To date, few studies have evaluated whether FMT is effective in IBS patients [16]. Furthermore, recent re- ports showed that the intestinal microbiome in the Japanese population is considerably different from those of other populations [17]. We already safely conducted FMT for Japanese patients with ulcerative colitis [18]. To clarify the efficacy of FMT in Japanese patients with IBS, we performed a single-arm, open-label, non-randomized study of FMT with metagenomic and psychological analyses.

\section{Materials and Methods}

\section{Ethics}

The Ethics Committee at Keio University School of Medicine approved the protocol (\#20130488), and all participants provided written informed consent. The study was registered at the University hospital Medical Information Network Center (UMIN000014617).

\section{Study Design}

In this single-center, open-label, non-randomized study, the safety and efficacy of FMT was evaluated in patients with refractory IBS who did not respond to medical therapy. Clinical followup was performed until 12 weeks after FMT.

\section{Participants}

Patients with IBS, eligible patients were 20 years or older with IBS diagnosed based on Rome III Diagnostic Criteria [19]. Patients with persistent symptoms despite medical therapy for at least a year were enrolled. Patients were excluded if they were pregnant or unable to give informed consent, and if they suffered severe liver or renal dysfunction. Patients who could not undergo endoscopy because of a psychiatric disorder were also excluded.

Donors, as we previously reported [18], healthy relatives within the second degree of relationship ( $\geq 20$ years of age) were screened using stool and serology analysis for bacterial, parasitic, and viral pathogens.

\section{FMT Procedure}

As we previously reported [20], donors were instructed to collect feces in an AneroPack ${ }^{\mathrm{TM}}$ (Mitsubishi Gas Chemical Co. Inc., Tokyo, Japan) and to bring the pack to the hospital at $4{ }^{\circ} \mathrm{C}$ on the day of the scheduled FMT. Approximately $100 \mathrm{~g}$ of feces was collected from donors, dissolved in $200 \mathrm{~mL}$ of saline, and filtered through a metal strainer to make liquid slurry. Fecal materials were administered to the patient via colonoscopy following standard bowel preparation.

\section{Clinical Outcomes}

The primary end point was a change in the Bristol stool form scale 4 weeks after FMT. Recovery to types 3-4 was considered a clinical response [21]. The secondary end point was a change in intestinal microbiota and psychiatric status during 12 weeks after FMT. Psychiatric status was assessed using the Hamilton Rating Scale for Depression (HAM-D) and Hamilton Anxiety Rating Scale (HAM-A) respectively by well-trained psychologist and/or psychiatrist in a blinded manner $[22,23]$. The study procedure is shown in Figure 1a.
Mizuno et al. 


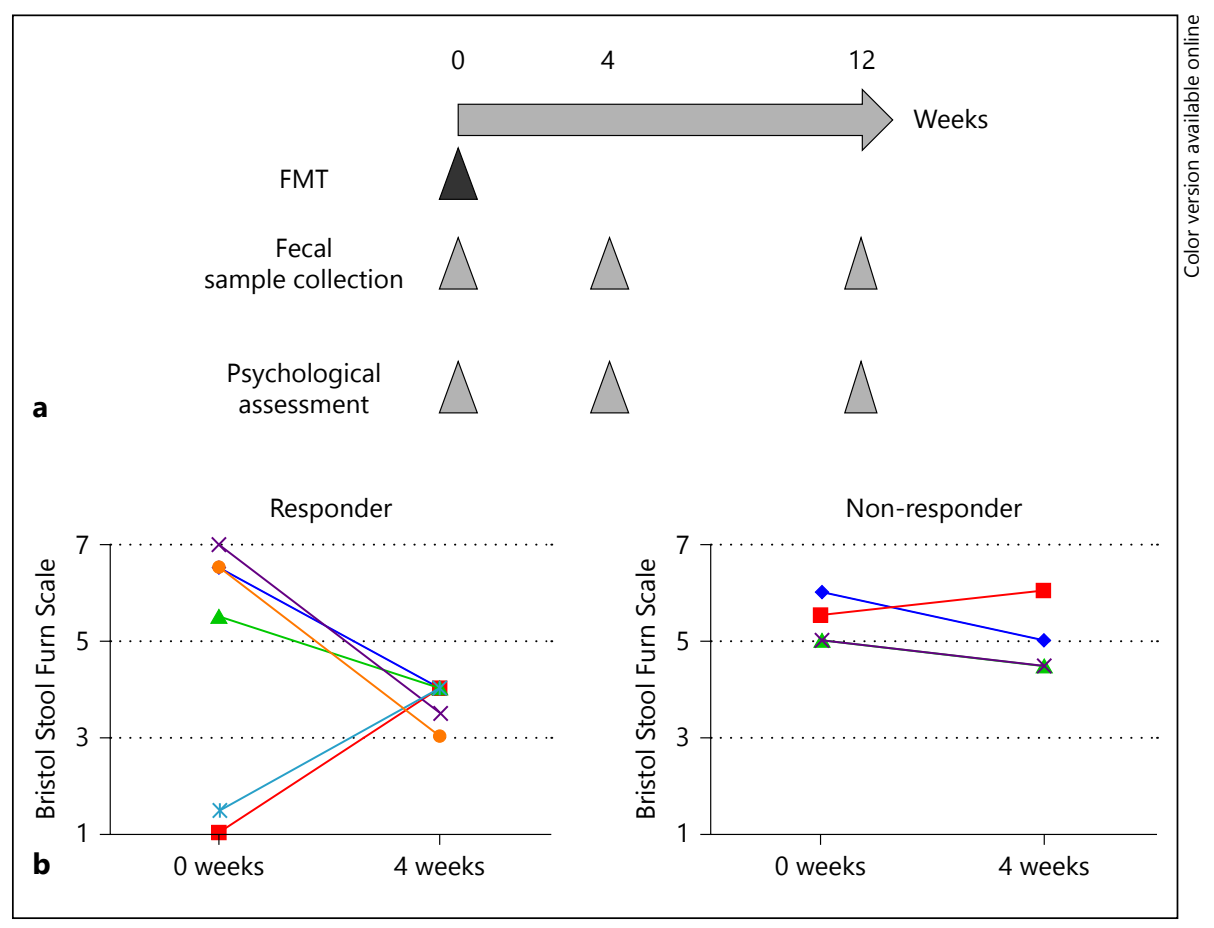

Fig. 1. a Overview of study procedure. b Change in stool form scale in responder and non-responder patients using the Bristol Stool Form Scale at baseline and at 4 weeks after FMT.

\section{Fecal Sample Collection}

Fecal samples were longitudinally collected from patients at weeks 0 , 4, and 12 after FMT, and from donors on the day of FMT. A total of 40 fecal samples were collected from patients and donors. The collected fresh feces were stored under anaerobic conditions in an AneroPack ${ }^{\mathrm{TM}}$ at $4^{\circ} \mathrm{C}$. The feces were frozen in $20 \%$ glycerol (Wako Pure Chemical Industries, Osaka, Japan)/phosphate-buffered saline solution (Life Technologies, Tokyo, Japan) by liquid nitrogen and stored at $-80^{\circ} \mathrm{C}$ until usage.

\section{Recovery of Bacteria from Fecal Samples and Bacterial}

DNA Extraction

Bacterial DNA was prepared as described previously [17]. In brief, bacterial DNA was extracted by enzymatic lysis using lysozyme (Sigma-Aldrich Co. LCC, Tokyo, Japan) and achromopeptidase (Wako). DNA samples were then purified by treatment with ribonuclease A (Wako), followed by precipitation with $20 \%$ polyethylene glycol solution (PEG6000 in $2.5 \mathrm{M}$ sodium chloride). DNA was then pelleted by centrifugation, rinsed with $75 \%$ ethanol, and dissolved in tris-ethylene diamine tetra acetic acid buffer.

\section{Sequencing and Processing of Bacterial 16S rRNA Genes from}

Fecal DNA

Multiplexed 16S amplicon sequencing was performed on the MiSeq Illumina platform; 16S rRNA gene V1-V2 region was amplified by PCR using the primers 27Fmod and 338R containing Illumina adaptor sequences and a unique 8-bp barcode. PCR amplicons were purified by AMPure XP magnetic purification beads (Beckman Coulter, Inc.), and quantified using the Quant-iT PicoGreen dsDNA Assay Kit. Equal amount of each PCR amplicon was mixed and then sequenced using MiSeq Reagent Kit v3 (600-cycles). Based on sample specific barcodes, obtained reads were assigned to each sample and paired-end reads were merged using fastq-join program. Reads with an average quality value $<25$, mismatches to both universal primers, and possible chimeric reads were removed. Among the high-quality reads, 3,000 reads per sample were randomly selected and grouped into operational taxonomic units by clustering using the UCLUST algorithm with a $96 \%$ identity threshold. Taxonomic assignments for each operational taxonomic unit were made by similarity search against the public $16 \mathrm{~S}$ and NCBI genome databases using the GLSEARCH program. For assignment at the phylum, family, genus, and species levels, sequence similarity thresholds of 70, 90, 94, and 96\% were employed, respectively. All the high-quality 16S V1-V2 sequences analyzed in this study were deposited into the DDBJ/GenBank/ EMBL database with accession number DRA005522. UniFrac distance and Principal coordinate analysis were used to assess the similarity of microbiota structures between each pair of samples.

\section{Statistical Analysis}

Results are expressed as the mean \pm SD. Groups of data were compared using the Student $t$ test. For multiple comparisons, statistical analysis was performed using the Kruskal-Wallis or oneway analysis of variance test depending on the distribution of the data. Post hoc analysis was performed by the Tukey-Kramer test. Differences were considered statistically significant when the $p$ value was $<0.05$. All analyses were conducted using GraphPad Prism software version 6 (GraphPad Software, San Diego, CA, USA).

\section{Results}

\section{Patient Enrollment}

We enrolled 10 patients from July 2014 through February 2016, comprising 8 patients with diarrhea-pre- 
Table 1. Patients' and donors' baseline characteristics. Disease type was defined according to Rome III diagnostic criteria

\begin{tabular}{lc}
\hline Patients' characteristics $(n=10)$ & \\
Age, years, median (range) & $40.1(25-57)$ \\
Sex, M:F, $n$ & $7: 3$ \\
Disease duration, years, median (range) & $13.9(5-40)$ \\
Disease type, $n(\%)$ & \\
$\quad$ IBS-D & $8(80)$ \\
IBS-C & $1(10)$ \\
IBS-M & $1(10)$ \\
\hline Donors' characteristics $(n=10)$ & \\
Age, years, median (range) & $52(23-79)$ \\
Sex, M:F, $n$ & $4: 6$ \\
Relationship, $n(\%)$ & \\
Spouse & $3(30)$ \\
Parent & $4(40)$ \\
Sibling & $3(30)$ \\
\hline
\end{tabular}

IBS-D, IBS with diarrhea; IBS-C, IBS with constipation; IBS-M, mixed IBS.

dominant IBS, 1 with constipation-predominant IBS, and 1 with mixed-type IBS. The patient and donor clinical characteristics are shown in Table 1.

\section{Donor Screening}

Screening of 11 healthy subjects for stool pathogens and serology resulted in 10 eligible donors. One patient was excluded because of positive screening results for infectious agents in feces. All donors donated feces that were used for infusion to patients. The mean age of donor tended to be higher than the age of the patient $(p=0.09)$. There was no significant difference in sex between donors and patients.

\section{Improvement of Stool form after FMT}

Six patients achieved a clinical response. The change in the stool form scale is shown in Figure 1b. The stool scale of 1 patient in the responder group worsened at week 12 , and no patients in the non-responder group achieved a clinical response at week 12 (online suppl. Fig. 1; for all online suppl.material,seewww.karger.com/doi/10.1159/000471919). There were no adverse events in this study.

These results suggested that our single FMT protocol had the potential to improve stool form in patients with IBS, but that the effect was sustained for a limited time.

\section{FMT Improved the Diversity of Intestinal Microbiota in Responders}

Intestinal microbial profiling was conducted by extracting genomic DNA from patient and donor fecal samples: 30 samples were collected from 10 patients and 10 samples were collected from 10 donors. The diversity of fecal microbiota in patients increased significantly at the 4 -week post-FMT evaluation ( $p=0.03$; Fig. $2 \mathrm{a}$ left), although there was no significant difference between donors and patients at baseline. Four weeks after FMT, the diversity of microbiota in responders was significantly higher than that of non-responders ( $p=0.02$; Fig. $2 \mathrm{a}$ right). Redundancy analysis of microbiota showed no significant change before and after FMT (Fig. 2b, c). These results suggested that there was a relationship between restoring the diversity of the microbiota in patients and the therapeutic effects of FMT.

\section{FMT Restored the Abundance of Bifidobacterium in Responders}

When comparing patient samples at baseline with samples from donors for responders (effective donors) and donors for non-responder (ineffective donors), taxonomic profiles showed that the phylum Firmicutes was dominant in almost all the donors and in patients with no significant difference. Of note, the abundance of the phylum Actinobacteria in effective donors was significantly higher than that of ineffective donors $(p=0.02)$ and patients $(p=0.01)$. Conversely, there was no significant difference between ineffective donors and patients (Fig. 3a). As shown in Figure 3b, the abundance of Actinobacteria in patients did not recover to the levels of donors at 4 weeks after FMT in the responder group, while it was much higher in donors compared with patients at baseline $(p=0.01)$. There was no significant difference in the non-responder group. Changes in the abundance of Actinobacteria between individuals demonstrated an upward trend after FMT in responders, but this was not seen in non-responders (online suppl. Fig. 2a). Changes in the abundance of Firmicutes between individuals showed a reverse trend (online suppl. Fig. 2b).

The abundance of the genus Bifidobacterium, which belongs to the phylum Actinobacteria, in effective donors was significantly higher than in ineffective donors $(p=$ $0.007)$ and patients $(p=0.02)$ but there was no significant difference between ineffective donors and patients (Fig. 3c). Figure 3d shows that the abundance of Bifidobacterium in patients did not recover to the levels of donors at 4 weeks after FMT in responders, while the abundance of Bifidobacterium in donors was significantly higher than in patients at baseline $(p=0.02)$. There was no significant difference among the 3 groups in the non-responders. Changes in the abundance of Bifidobacterium demonstrated an upward trend after FMT in responders, but were not seen in non-responders (online suppl. Fig. 2c).
Mizuno et al. 
Fig. 2. a Left: microbiota diversity (Shannon index) of 10 donors and 10 patients before and at 4 weeks after FMT. Right: microbiota diversity of patients in responders $(n=$ $6)$ and non-responders $(n=4)$ before and at 4 weeks after FMT. b Redundancy analysis of microbiota from donors and patients before and at 4 weeks after FMT in responders and non-responders with weighted UniFrac analysis. c Redundancy analysis of microbiota from donors and patients before and at 4 weeks after FMT in responders and non-responders with unweighted UniFrac analysis.

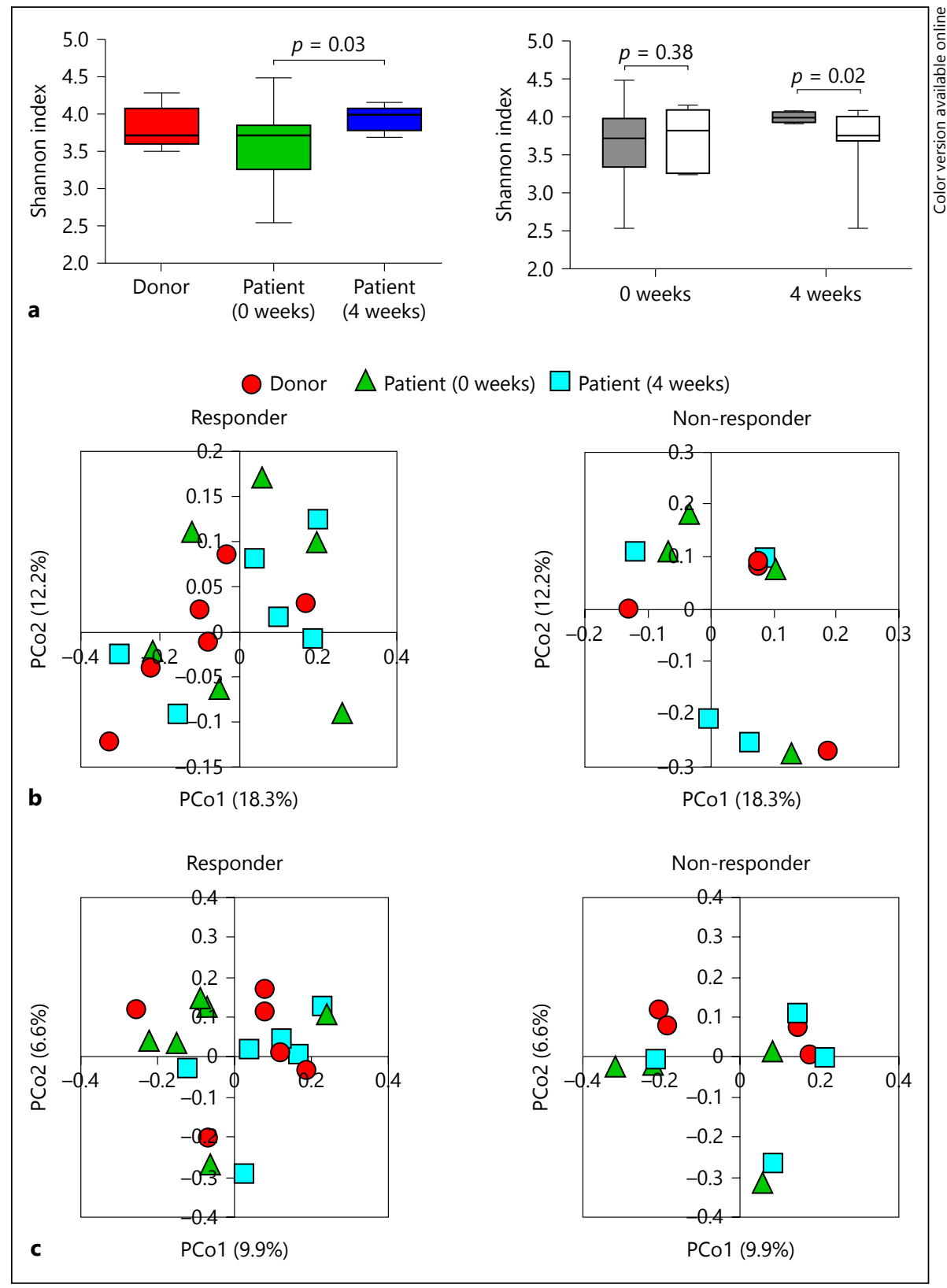

These results indicated that the abundance of the genus Bifidobacterium in donor feces was related to the therapeutic efficacy of FMT, but the increase in Bifidobacterium in patients did not correlate with the therapeutic efficacy of FMT.

\section{FMT Improved Psychiatric Status and Abdominal} Symptom in Both Responders and Non-Responders

Patients with IBS often suffer from psychiatric symptoms [24]. Thus, HAM-D before and at 4 and 12 weeks after FMT was assessed. Psychological assessments for 1 responder and 1 non-responder were not done. The HAM-D score significantly improved at 4 week after FMT $(p=0.01)$, but returned to the baseline level at 12 weeks (Fig. 4a left). HAM-A was also assessed and was improved at 4 weeks after FMT, but there was no significant difference ( $p=0.06$; Fig. $4 \mathrm{~b}$ left). Intriguingly, change in HAM-D and HAM-A scores showed no significant difference between responders and non-responders (Fig. 4a, $\mathrm{b}$ right), but gastrointestinal symptoms including abdominal pain after FMT evaluated with HAM-A were significantly improved in responders, not in non-respond- 


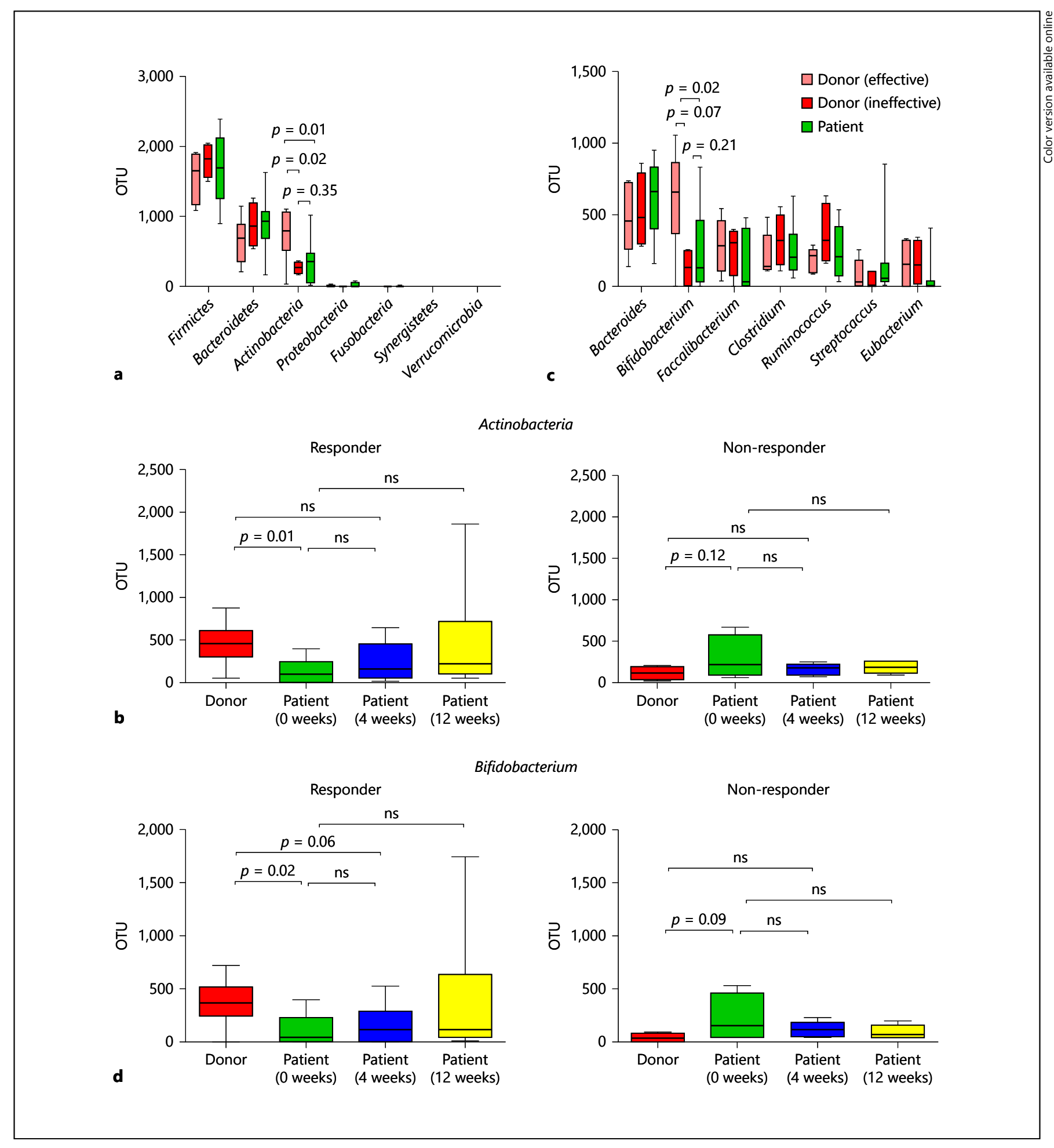

Fig. 3. Analysis of the microbiome. a Comparison of 7 fecal bacteria at the phylum level in effective donors $(n=6)$, ineffective donors $(n=4)$ and all patients $(n=10)$. b Abundance of Actinobacteria in donors and patients before and at 4 and 12 weeks after FMT in responders $(n=6)$ and non-responders $(n=4)$. c Comparison of the top 7 fecal bacteria at the genus level in effective donors $(n=6)$, ineffective donors $(n=4)$ and all patients $(n=$ $10)$. d Abundance of Bifidobacterium in donors and patients before and at 4 and 12 weeks after FMT in responders $(n=6)$ and non-responders $(n=4)$. Average OTU abundance is shown for each group. OTU, operational taxonomic unit; ns, not significant. 
Fig. 4. Analysis of patients' psychological status and abdominal symptoms. a HAM-D score of patients at baseline and at 4 and 12 weeks after FMT in 8 patients: left: in responders $(n=5)$ and right: non-responders $(n=3)$. b HAM-A score of patients at baseline and at 4 and 12 weeks after FMT in 8 patients: left: responders $(n=5)$ and right: nonresponders $(n=3)$. c Gastrointestinal symptoms evaluated in HAM-A of patients at baseline and 12 weeks after FMT in 8 patients: left: responders $(n=5)$ and right: nonresponders $(n=3)$.

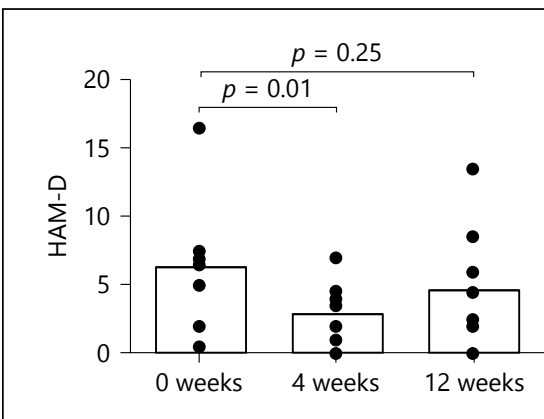

a
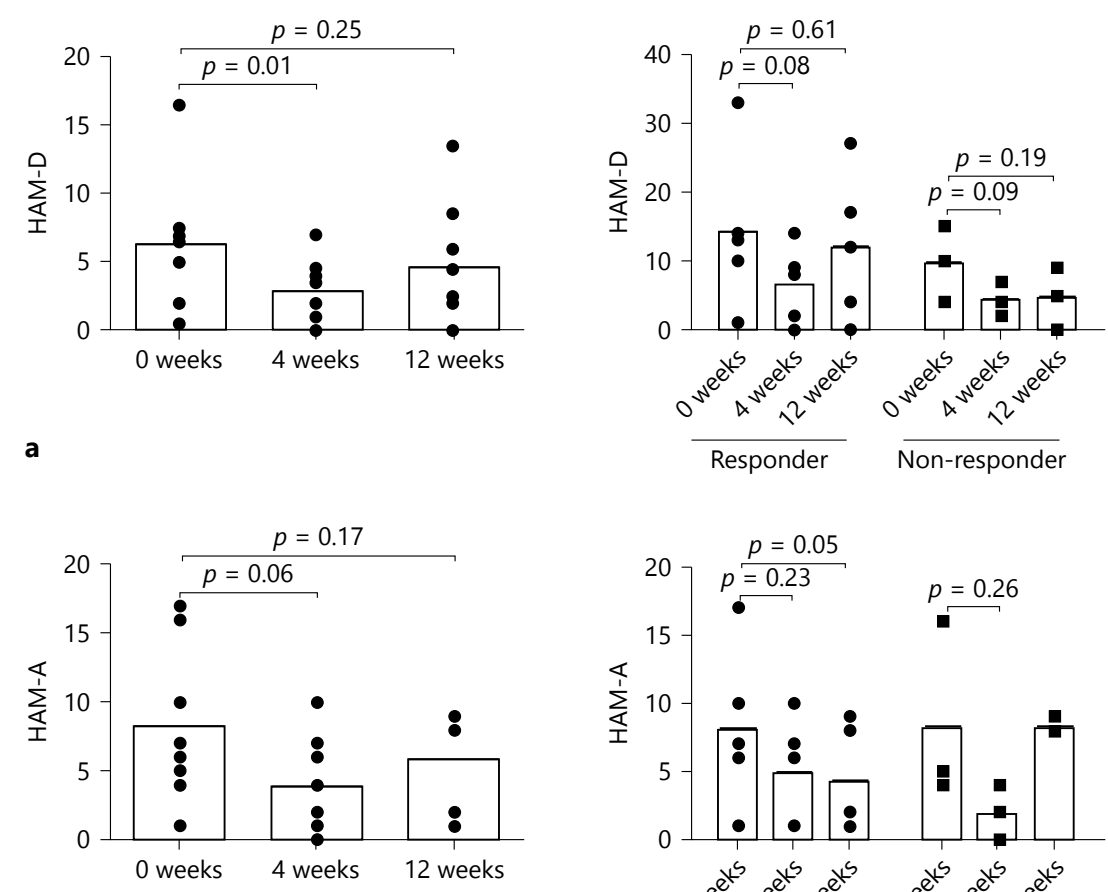

b

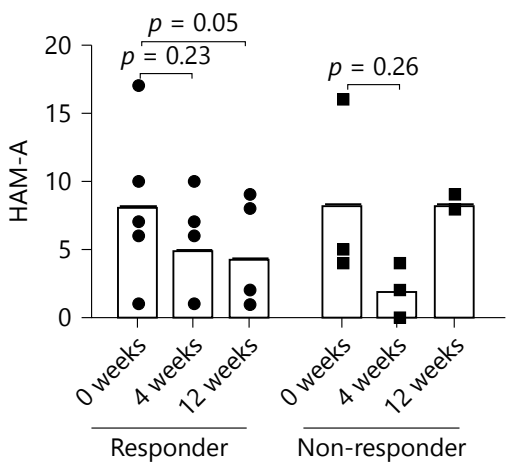

ers (Fig. 4c; online suppl. Fig. 3a). We also analyzed the changes in abundance of Actinobacteria of each patient at baseline and at 4 weeks after FMT. Effective patients were defined as patients achieved improvement in gastrointestinal symptom, but there is no significant correlation between the changes in abundance of Actinobacteria and the improvement of gastrointestinal symptoms after FMT (online suppl. Fig. 3b).

These results suggest that FMT has a therapeutic effect for IBS patients' psychiatric status irrespective of the presence or absence of stool abnormalities, but the effect may not be sustained for 12 weeks.

Fecal Microbiota Transplantation for IBS

\section{Discussion}

This open-label pilot study demonstrated that FMT for patients with IBS was conducted safe, and relatively effective; 6 of 10 patients recovered to types 3-4 of stool score at 4 weeks after FMT. The metagenomic analysis in this study revealed that (1) the decreased diversity of microbiota in patients before FMT was recovered to the level of the donor after FMT, and (2) the efficacy of FMT was clearly dependent on the status of increased Actinobacteria phylum (especially Bifidobacterium genus) in donor feces, but not on changes in the composition of patient 
microbiota before or after FMT. Surprisingly, we also found that FMT significantly improved the psychological status after FMT irrespective of IBS responders and nonresponders.

This is the first study to analyze the composition of intestinal microbiota in donors and patients and the change in microbiota composition before and after FMT in IBS patients. The number of enrolled patient was small but sufficient for the first registered trial to evaluate the efficacy of FMT in addition to the safety profile in IBS patients.

In this study, we attempted to extend the application of FMT for intractable intestinal diseases. The results of 2 RCT for ulcerative colitis patients were controversial $[25,26]$. In one positive RCT, researchers used feces from one specific healthy individual after they observed that the specific donor feces were effective in an on-going study, suggesting the concept of a "super-donor" for successful FMT. Similarly, in this study, we found that one of the positive predictors for the efficacy of FMT for IBS patients was a donor factor; specific donors that contained high amounts of the Bifidobacterium genus were effective for IBS patients. This suggests that FMT using specific donor feces rich in Bifidobacterium genus may stimulate patient microbiota to recover from decreased diversity to the microbiota level of the donor. Although further studies are warranted, these data suggest that the selection of donor feces is a crucial factor for the success of FMT. A recent study showed that the stimulation, but not replacement, of recipient microbiota by donor feces was important as a therapeutic mechanism of FMT [27]. Taken together with the findings of our current study, fecal microbiota "stimulation" of recipient microbiota by donor feces, but not fecal microbiota "transplantation" of donor feces, seems to be an appropriate term for fecal microbiota transfer protocols that are currently used.

In addition to intestinal diseases, recent studies have shown that gut microbiota in patients with some extraintestinal disorders differed from that of healthy individuals. One placebo-controlled RCT of FMT in metabolic syndrome patients showed that insulin sensitivity was significantly increased after FMT [28]. Moreover, another group performed a pilot study of FMT in 4 patients with acute graft-vs.-host disease receiving bone marrow transplantation [29]. Previous studies showed that changes in intestinal microbiota altered patients' psychiatric status [30], and modulation of the relationship between stress and intestinal microbiota composition altered brain functions [31]. A recent study sug- gested that Bifidobacterium counts in the gut microbiota of patients with major depressive disorder were significantly decreased compared with those of healthy volunteers [32]. A randomized controlled trial was conducted on the effect of probiotics on the neurophysiological parameters of patients with autism spectrum disorders [33]. There have also been some case reports of FMT for neurogenic disorders [34]. Our results suggested that FMT for IBS patients has the potential to improve their psychiatric status. A previous study demonstrated that patients with diarrhea-predominant IBS and depression had similar alterations in fecal microbiota. Together with our study, these results suggest that FMT may be effective for patients with depression. Furthermore, short-chain fructo-oligosaccharides significantly reduced anxiety scores and increased fecal Bifidobacterium [35]. We showed that the abundance of Bifidobacterium tended to increase after FMT in responders. Furthermore, the improved stool form of patients with IBS was associated with the increase in Bifidobacterium. We also showed that the decreased diversity of microbiota in patients before FMT recovered to the level of the donor after FMT. These data suggest that Bifidobacterium-rich donor feces may stimulate the growth of minor strains in patient microbiota that were undetectable before FMT to increase their diversity after FMT.

In addition to analysis of abdominal symptoms, we assessed the psychiatric status of patients. Surprisingly, the HAM-D score was decreased in 7 of 8 patients at 4 weeks after FMT irrespective of whether they responded to FMT. This indicated that the improved clinical symptoms of IBS patients were not caused by the placebo effect but a change in intestinal microbiota. The therapeutic effect of FMT on patients with psychological disorders was sustained for a limited time, up to 4 weeks after FMT, and the abundance of Bifidobacterium tended to decrease from 4 to 12 weeks after FMT (online suppl. Fig. 2c). This result indicated that the improvement of abdominal symptoms of patients with IBS depended on intestinal microbial change caused by FMT.

In conclusion, this study demonstrated that FMT could improve of the stool form and depressed mood and/or anxiety via alterations in intestinal microbiota. Notably, Bifidobacterium-rich donor feces may stimulate minor strains of the patient microbiota growth that were undetectable before FMT to increase their diversity after FMT. Although large-scale RCTs are required, the current study highlights the potential of FMT as a therapeu-
36

Digestion 2017;96:29-38

DOI: $10.1159 / 000471919$
Mizuno et al. 
tic option for patients with IBS as well as psychiatric disorders such as major depressive disorders and anxiety disorders.

\section{Acknowledgments}

The authors thank K. Hirata, H. Kiyohara, T. Yamane, and K. Mori (Keio University) for technical assistance. The authors also thank K. Nanki, Y. Nakazato, H. Ogata, and Y. Iwao (Keio University) for helpful discussion and K. Matsuoka (Tokyo Medical and Dental University) for establishing the FMT procedure.

\section{Disclosure Statement}

All authors declare no conflicts of interest.

\section{Funding}

This study was supported in part by Health and Labor Sciences Research Grantsfor research on intractable diseasesfrom the Japanese Ministry of Health (T.K.), a Grant-in-Aid for Scientific Research C (25460301, T.M.), Takeda Science Foundation (S.M.), Keio Gijuku Academic Development Funds (T.M.), and Keio University Grantin-Aid for Encouragement of Young Medical Scientists (S.M.).

\section{References}

1 Gralnek IM, Hays RD, Kilbourne A, Naliboff B, Mayer EA: The impact of irritable bowel syndrome on health-related quality of life. Gastroenterology 2000;119:654-660.

2 Simren M, Svedlund J, Posserud I, Bjornsson ES, Abrahamsson H: Health-related quality of life in patients attending a gastroenterology outpatient clinic: functional disorders versus organic diseases. Clin Gastroenterol Hepatol 2006;4:187-195.

3 Kassinen A, Krogius-Kurikka L, Makivuokko $\mathrm{H}$, Rinttila T, Paulin L, Corander J, Malinen E, Apajalahti J, Palva A: The fecal microbiota of irritable bowel syndrome patients differs significantly from that of healthy subjects. Gastroenterology 2007;133:24-33.

4 Krogius-Kurikka L, Lyra A, Malinen E, Aarnikunnas J, Tuimala J, Paulin L, Makivuokko H, Kajander K, Palva A: Microbial community analysis reveals high level phylogenetic alterations in the overall gastrointestinal microbiota of diarrhoea-predominant irritable bowel syndrome sufferers. BMCGastroenterol 2009;9:95.

5 Barbara G, Feinle-Bisset C, Ghoshal UC, Quigley EM, Santos J, Vanner S, Vergnolle N, Zoetendal EG: The intestinal microenvironment and functional gastrointestinal disorders. Gastroenterology 2016;pii:S001 6-5085(16)00219-5.

6 Simren M, Barbara G, Flint HJ, Spiegel BM, Spiller RC, Vanner S, Verdu EF, Whorwell PJ, Zoetendal EG; Rome Foundation Committee: Intestinal microbiota in functional bowel disorders: a Rome foundation report. Gut 2013; 62:159-176

7 Mayer EA, Savidge T, Shulman RJ: Brain-gut microbiome interactions and functional bowel disorders. Gastroenterology 2014; 146: 1500-1512.

8 Mayer EA, Tillisch K, Gupta A: Gut/brain axis and the microbiota. J Clin Invest 2015;125: 926-938.

9 Hungin AP, Becher A, Cayley B, Heidelbaugh JJ, Muris JW, Rubin G, Seifert B, Russell A, De Wit NJ: Irritable bowel syndrome: an integrated explanatory model for clinical practice. Neurogastroenterol Motil 2015;27:750-763.
10 O'Mahony L, McCarthy J, Kelly P, Hurley G, Luo F, Chen K, O’Sullivan GC, Kiely B, Collins JK, Shanahan F, Quigley EM: Lactobacillus and bifidobacterium in irritable bowel syndrome: symptom responses and relationship to cytokine profiles. Gastroenterology 2005; 128:541-551.

11 Halmos EP, Power VA, Shepherd SJ, Gibson PR, Muir JG: A diet low in FODMAPs reduces symptoms of irritable bowel syndrome. Gastroenterology 2014;146:67-75.e5.

12 Tuck CJ, Muir JG, Barrett JS, Gibson PR: Fermentable oligosaccharides, disaccharides, monosaccharides and polyols: role in irritable bowel syndrome. Expert Rev Gastroenterol Hepatol 2014;8:819-834.

13 McIntosh K, Reed DE, Schneider T, Dang F, Keshteli AH, De Palma G, Madsen K, Bercik P, Vanner S: FODMAPs alter symptoms and the metabolome of patients with IBS: a randomised controlled trial. Gut 2016;pii:gutjnl-2015-311339.

14 van Nood E, Vrieze A, Nieuwdorp M, Fuentes S, Zoetendal EG, de Vos WM, Visser CE, Kuijper EJ, Bartelsman JF, Tijssen JG, Speelman P, Dijkgraaf MG, Keller JJ: Duodenal infusion of donor feces for recurrent Clostridium difficile. N Engl J Med 2013;368:407-415.

15 Seekatz AM, Aas J, Gessert CE, Rubin TA, Saman DM, Bakken JS, Young VB: Recovery of the gut microbiome following fecal microbiota transplantation. MBio 2014;5:e00893e00894.

16 Pinn DM, Aroniadis OC, Brandt LJ: Is fecal microbiota transplantation the answer for irritable bowel syndrome? A single-center experience. Am J Gastroenterol 2014;109:18311832.

17 Nishijima S, Suda W, Oshima K, Kim SW, Hirose Y, Morita H, Hattori M: The gut microbiome of healthy Japanese and its microbial and functional uniqueness. DNA Res 2016; 23:125-133.

18 Mizuno S, Nanki K, Matsuoka K, Saigusa K, Ono K, Arai M, Sugimoto S, Kiyohara H, Nakashima M, Takeshita K, Naganuma M, Suda W, Hattori M, Kanai T: Single fecal microbi- ota transplantation failed to change intestinal microbiota and had limited effectiveness against ulcerative colitis in Japanese patients. Intest Res 2017;15:68-74.

19 Longstreth GF, Thompson WG, Chey WD, Houghton LA, Mearin F, Spiller RC: Functional bowel disorders. Gastroenterology 2006;130:1480-1491.

20 Matsuoka K, Mizuno S, Hayashi A, Hisamatsu T, Naganuma M, Kanai T: Fecal microbiota transplantation for gastrointestinal diseases. Keio J Med 2014;63:69-74.

21 Lewis SJ, Heaton KW: Stool form scale as a useful guide to intestinal transit time. Scand J Gastroenterol 1997;32:920-924.

22 Hamilton M: The assessment of anxiety states by rating. Br J Med Psychol 1959;32:50-55.

23 Hamilton M: A rating scale for depression. J Neurol Neurosurg Psychiatry 1960;23:56-62.

24 Lackner JM, Gudleski GD, Thakur ER, Stewart TJ, Iacobucci GJ, Spiegel BM: The impact of physical complaints, social environment, and psychological functioning on IBS patients' health perceptions: looking beyond GI symptom severity. Am J Gastroenterol 2014; 109:224-233.

25 Moayyedi P, Surette MG, Kim PT, Libertucci J, Wolfe M, Onischi C, Armstrong D, Marshall JK, Kassam Z, Reinisch W, Lee CH: Fecal microbiota transplantation induces remission in patients with active ulcerative colitis in a randomized controlled trial. Gastroenterology 2015;149:102-109.e6.

26 Rossen NG, Fuentes S, van der Spek MJ, Tijssen JG, Hartman JH, Duflou A, Lowenberg M, van den Brink GR, Mathus-Vliegen EM, de Vos WM, Zoetendal EG, D'Haens GR, Ponsioen $\mathrm{CY}$ : Findings from a randomized controlled trial of fecal transplantation for patients with ulcerative colitis. Gastroenterology 2015;149:110-118.e4.

27 Li SS, Zhu A, Benes V, Costea PI, Hercog R, Hildebrand F, Huerta-Cepas J, Nieuwdorp M, Salojarvi J, Voigt AY, Zeller G, Sunagawa S, de Vos WM, Bork P: Durable coexistence of donor and recipient strains after fecal microbiota transplantation. Science 2016;352:586-589. 
28 Vrieze A, de Groot PF, Kootte RS, Knaapen M, van Nood E, Nieuwdorp M: Fecal transplant: a safe and sustainable clinical therapy for restoring intestinal microbial balance in human disease? Best Pract Res Clin Gastroenterol 2013;27:127-137.

29 Kakihana K, Fujioka Y, Suda W, Najima Y, Kuwata G, Sasajima S, Mimura I, Morita H, Sugiyama D, Nishikawa H, Hattori M, Hino Y, Ikegawa S, Yamamoto K, Toya T, Doki N, Koizumi K, Honda K, Ohashi K: Fecal microbiota transplantation for patients with steroid-resistant acute graft-versus-host disease of the gut. Blood 2016;128:2083-2088.

30 Mangiola F, Ianiro G, Franceschi F, Fagiuoli S, Gasbarrini G, Gasbarrini A: Gut microbiota in autism and mood disorders. World J Gastroenterol 2016;22:361-368.
31 Wong ML, Inserra A, Lewis MD, Mastronardi CA, Leong L, Choo J, Kentish S, Xie P, Morrison $M$, Wesselingh SL, Rogers GB, Licinio J: Inflammasome signaling affects anxiety- and depressive-like behavior and gut microbiome composition. Mol Psychiatry 2016;21:797805.

32 Aizawa E, Tsuji H, Asahara T, Takahashi T, Teraishi T, Yoshida S, Ota M, Koga N, Hattori $\mathrm{K}$, Kunugi $\mathrm{H}$ : Possible association of Bifidobacterium and Lactobacillus in the gut microbiota of patients with major depressive disorder. J Affect Disord 2016;202:254257.

33 Santocchi E, Guiducci L, Fulceri F, Billeci L, Buzzigoli E, Apicella F, Calderoni S, Grossi E, Morales MA, Muratori F: Gut to brain interaction in Autism Spectrum Disorders: a ran- domized controlled trial on the role of probiotics on clinical, biochemical and neurophysiological parameters. BMC Psychiatry 2016; 16:183.

34 Xu MQ, Cao HL, Wang WQ, Wang S, Cao XC, Yan F, Wang BM: Fecal microbiota transplantation broadening its application beyond intestinal disorders. World J Gastroenterol 2015;21:102-111.

35 Azpiroz F, Dubray C, Bernalier-Donadille A, Cardot JM, Accarino A, Serra J, Wagner A, Respondek F, Dapoigny M: Effects of scFOS on the composition of fecal microbiota and anxiety in patients with irritable bowel syndrome: a randomized, double blind, placebo controlled study. Neurogastroenterol Motil 2017; 29. 